\title{
On the Semantics of Exclusion and Inclusion Phrases
}

\author{
R. Zuber \\ CNRS, Paris
}

\section{Introduction}

Exclusion constructions, as I understand this term here and which have sometimes been called also exception or exceptive constructions, are constructions containing exclusion noun phrases. Similarly with inclusion constructions. Exclusion noun phrases, EXCL phrases henceforth, are italicised NPs in (1) and (2) and inclusion noun phrases, INCL phrases henceforth, are exemplified in (3):
a. Every student except /but Leo and Lea was sleeping
b. Every student except/but five passed the examination
c. Every student except/but Albanians is drinking
a. No student but Leo and Lea was at the movie
b. Lea danced with no student but one
c. No animal except the cat is dangerous
a. All/möst/somelfive students, including Leo visited the museum
b. All/most/somelfive students, including the five in the back passed the examination
c. All /most/some animals, including cats, are dangerous

So on the surface EXCL phrases have the general form NP connect E, where connect is a binary connective denoting but/exceptt in the case of EXCL phrases and including in the case of INCL phrases. Since there is no danger of confusion I will speak indistinguishibly about the syntactic or semantic arguments of connect. As the above examples show, the expression $\mathbf{E}$, considered as a syntactic argument of connect, can be a proper noun, a conjunction of proper nouns, a numeral, an adjective, and, as we will see, certain quantified noun phrases and possibly a bare noun phrase. The characterisation of the semantic type of connect and of its second argument $\mathbf{E}$, independently of what is instanciated, and more generally the semantic status of these two expressions is the main purpose of this paper. I will claim, contrary to many other proposals, that connect in EXCL as well in INCL phrases should be viewed as connecting (nominal) determiners.

I will be mainly interested in the connective except which will be called the exclusion connective. This denomination should not be taken, however, as always implying the exclusion meaning in all noun phrases since, as I will show, in some, in fact many, languages, some exclusion connectives also have the additive meaning. The connective including will be called inclusion connective.

In addition to INLC phrases containing explicitly a connective as illustrated in (3), and which can be called positive inclusion phrases one also finds negative inclusion phrases, without any explicit binary connective on the surface, as in (4):
a. No student/few students, not even Leo
b. No student, not even one
c. No student, not even Albanians 
In spite of the fact that there are many recent works concerning EXCL phrases (cf. von Fintel 1993, Hoeksema 1995, Keenan 1993, Moltmann 1995, 1996, Zuber 1997) the interest in EXCL phrases and sentences containing them is not recent since they were an object of study for many medieval logicians (cf. Kretzman 1982). This is not exactly the case with INCL phrases. In this paper I will try to provide a unifying, if not a unified, semantic analyse of both types of phrases. I want in particular to point out to various similarities in both types of phrases and to suggest a way of accounting for a possible semantic relationship between an exclusion phrase and the corresponding inclusion phrase.

There are strong constraints, often semantic in nature, on which NPs can occur as arguments of connectives in EXCL and INCL phrases. A general constraint concerning both EXCL and INCL phrases is the the first argument in each case must be a quantified noun phrase (QNP). Thus the examples in (5) are impossible:
a. *Leo and Lea except Leo
b. *Leo and Lea and Sue, including two students
c. *Leo and Lea, including Leo

In addition not all quantified noun phrases can take the position of the first argument. In the case of EXCL phrases this additional constraint on the first argument is easily expressed: only universally quantified noun phrases can be first arguments in exclusion phrases: so we can have noun phrases which denote either positive universal quantifiers as in (1) or negative universal quantifiers as in (2). Concerning the first argument of the connective including in INCL phrases it also must be a QNP but with various additional properties. In particular that quantifiers denoted by these NPs cannot be monotonically decreasing with respect to the first argument. Thus the following noun phrases are not acceptable:
a. * No student, including Leo
b. ?Few students, including Leo
c. *At most three students, including Leo

Less known are constraints on the second argument of connect in EXCL and INCL phrases. As the examples given above show it can be a proper name or a conjunction of proper names. It can also be a bare numeral determiner. It can also be a common noun or an adjective but in this case it is usually an elliptic form of a QNP. There are, however, also various restrictions which remain to be enumerated if not explained: the grammatical status of the following examples is not clear:

a. ?Every student, except most Albanian ones

b. ?No student except some Albanian ones

On the other hand the inclusion connective seems to admit much larger range of second arguments since the examples similar to those in (7) are in this case acceptable:

a. Most students, including most Albanian ones

b. All students, including some Albanian ones

There is an interesting opposition between EXCL phrases and INCL phrases concerning the occurence as the second argument of the noun phrases such as at least $n$ and at most $n$. These noun phrases seem to occur in a complementary way in EXCL and INCL phrases. Thus compare the following examples: 
a. Most/all students, including at least 5 Albanians

b. *Most/all students, including at most 5 Albanians

a. All students, eccept at most 5 (Albanians)

b. *All students, except at least 5 Albanians

The last difference in behaviour of ECXL and INCL phrases with respect to the second argument I mention will be taken into account in my proposal. It concerns the semantic specificity (or definitness) of this argument. One observes that INCL phrases, as opposed to EXCL ones do not accept bare numeral determiners as the second argument:

(11) a. *Most students, including five

b. Most students, including the five (at the back)

c. All students except five

I mention these relatively complex data in order to partially justify the fact that I am not able to study all semantic aspects of EXCL and INCL phrases. I am mainly interested in providing a semantics for such phrases independently of the constraints which govern their production. As we will see, the description I will offer will be fully clear, first of all, for "atomic" phrases, that is those which denote atoms, in the technical sense, of the corresponding denotational algebras.

\section{Formal preliminaries}

The theoretical tools which will be used are those of by now standard formal semantics as used in the generalized quantifiers theory enriched by the Boolean semantics as developed by Keenan (Keenan 1983, Keenan and Faltz 1985). This means in particular that all logical types $\mathbf{D}_{\mathbf{C}}$, denotations of the category $\mathbf{C}$, form atomic (and complete) Boolean algebras. The meet operation in any Boolean algebra will be noted, ambiguously, by and. The partial order defined in these denotational algebras is interpreted as a generalized entailment, (entailment, for short). Thus it is meaningful to say that an entailment holds between two NPs or between two nominal determiners, etc. For instance given this framework we can (truthfully) say that the NP in (12a) entails the NP in (12b) and in (12c) and that the determiner in (13a) entails the determiner in (13b):

(12) a. All students except the Albanian ones

b. No Albanian student

c. Not all students

a. No...except twelve

b. twelve

So we need algebras in which NPs denote and different, but related, algebras in which nominal determiners denote. Noun phrases denote in the algebra $\mathbf{D}_{\mathbf{N P}}$ of functions from properties onto truth values. This means that elements of $\mathbf{D}_{\mathbf{N P}}$ are sets of properties. Denotations of nominal determiners, dets for short, are those functions from properties onto a set of properties which satisfy the property of conservativity in the sense that if $\mathbf{D}$ is a function denoted by a determiner then for all properties $\mathbf{X}$ and $\mathbf{Y}, \mathbf{D}(\mathbf{X})(\mathbf{Y})$ is true iff $\mathbf{D}(\mathbf{X})(\mathbf{X} \cap \mathbf{Y})$ is true. For any property $\mathbf{P}$ and any det $\mathbf{D}$ we define $\mathbf{D}_{\mathbf{P}}$, the det $\mathbf{D}$ restricted by $\mathbf{P}$, a $\mathbf{S}$ 
$\mathbf{D}_{\mathbf{P}}(\mathbf{X})=\mathbf{D}(\mathbf{P} \cap \mathbf{X})$. Dets restricted by a property are denotations of some pseudonoun phrases like Albanian ones occuring for instance in (12a). Trivially any det is restricted by the universal property 1 , the unit of the algebra of properties.

Dets, and corresponding type $<1>$ quantifiers have two negations: prefixenegation not-D, corresponding to the Boolean complement $\mathbf{D}^{\prime}$ of $\mathbf{D}$ and postnegation D-not defined by the following condition: $\mathbf{D}-\operatorname{not}(\mathbf{X})(\mathbf{Y})$ is true iff $\mathbf{D}(\mathbf{X})\left(\mathbf{Y}^{\prime}\right)$ is true, where $\mathbf{Y}^{\prime}$ is the Boolean complement of $\mathbf{Y}$.

There are two important sub-classes of conservative functions: intersective functions, INT, and co-intersective functions, CO-INT (Keenan 1993). Let the sentence DetCNVP denote the proposition $\mathbf{D}(\mathbf{S})(\mathbf{P})$, where $\mathbf{D}$ is the denotation of Det, $\mathbf{S}$ is the denotation of the common noun $\mathbf{C N}$ and $\mathbf{P}$ is the denotation of the VP. So the sentence DetCNVP is true iff the property $\mathbf{P}$ belongs to the set $\mathbf{D}(\mathbf{S})$. Now a function $\mathbf{F}$ is intersective, $\mathbf{F} \in \mathbf{I N T}$, iff for all properties $\mathbf{X}, \mathbf{Y}, \mathbf{Z}$ and $\mathbf{W}$, if $\mathbf{X} \cap \mathbf{Y}=\mathbf{Z} \cap \mathbf{W}$ then $\mathbf{F}(\mathbf{X})(\mathbf{Y})$ is true iff $\mathbf{F}(\mathbf{Z})(\mathbf{W})$ is true. Similarly, $\mathbf{F} \in \mathbf{C O}$-INT iff for all properties $\mathbf{X}, \mathbf{Y}, \mathbf{Z}$ and $\mathbf{W}$, if $\mathbf{X}-\mathbf{Y}=\mathbf{Z}-\mathbf{Y}$ then $\mathbf{F}(\mathbf{X})(\mathbf{Y})$ is true iff $\mathbf{F}(\mathbf{Z})(\mathbf{W})$ is true. It is easy to see from these definitions that intersective and cointersective functions are related by the post-negation. We have the following equivalence: F $\in$ INT iff F-not $\in$ CO-INT. Both sets INT and CO-INT form atomic (and complete) Boolean algebras with the Boolean operations defined pointwise. Atoms of INT are functions at $\mathbf{P}$, where $\mathbf{P}$ is a property, such that $\operatorname{at}_{\mathbf{P}}(\mathbf{X})(\mathbf{Y})$ is true iff $\mathbf{X} \cap \mathbf{Y}=\mathbf{A}$. Similarly atoms of CO-INT are functions at $\mathbf{P}$ such that $\operatorname{at}_{\mathbf{P}}(\mathbf{X})(\mathbf{Y})$ is true iff $\mathbf{X}-\mathbf{Y}=\mathbf{P}$.

Notice that many determiners found in ECXL phrases denote atoms of INT or CO-INT. For instance let the common noun student denote the property $\mathbf{S}$, the verb phrase danced denote the property $\mathrm{D}$, the common noun Albanians denote the property $\mathbf{A}$ and the proper name Leo denotes the (atomic) property $\{\mathbf{L}\}$. Consider now the following sentences:

a. No student except Albanians danced

b. Every student except Leo danced

One can easily check that sentence (14a) is true iff $\mathbf{S} \cap \mathbf{D}=\mathbf{A}$ and sentence (14b) is true iff $\mathbf{S}-\mathbf{D}=\{\mathbf{L}\}$. So we can say that both these sentences contain "atomic" determùiners from which EXCL phrases are formed.

Both algebras, INT and CÖ-INT are isomorphic to the algebra $\mathbf{D}_{\mathbf{N P}}$ of possible denotations of noun phrases (cf. Keenan 1993). This means in patricular that there is one to one correspondence between for instance functions denoted by negatiive universally quantified determiners and noun phrases and in adition this correspondence associates atoms of one algebra with atoms of the other algebra. For instance from the fact that noun phrases given in (15) are equivalent and that (15a) is formed from an atomic determiner, we conclude on the basis of the indicated isomorphism that $(15 \mathrm{~b})$ is an atomic noun phrase:
a. No existent except Leo

b. Only Leo

Furthermore, both algebras, INT and CO-INT contain, in the technical sense, the following (atomic and complet) sub-algebras: cardinal intersective algebra, CINT and cardinal co-intersective algebra CC-INT, respectively. These algebras 
are defined as follows: $\mathbf{F} \in \mathbf{C I N T}$ iff for all properties $\mathbf{X}, \mathbf{Y}, \mathbf{Z}$ and $\mathbf{W}$, if $\operatorname{card}(X \cap Y)=\operatorname{card}(Z \cap W)$ then $F(X)(Y)$ is true iff $F(Z)(W)$ is true. Analogously for co-intersective cardinal algebras: $\mathbf{F} \in \mathbf{C C}$-INT iff for all properties $\mathbf{X}, \mathbf{Y}, \mathbf{Z}$ and $\mathbf{W}$, if $\operatorname{card}(\mathbf{X}-\mathbf{Y})=\operatorname{card}(\mathbf{Z}-\mathbf{W})$ then $\mathbf{F}(\mathbf{X})(\mathbf{Y})$ is true iff $\mathbf{F}(\mathbf{Z})(\mathbf{W})$ is true. For any cardinal $\mathbf{c}$, the functions $\mathbf{f}_{\mathbf{c}}$ such that $\mathbf{f}_{\mathbf{c}}(\mathbf{X})(\mathbf{Y})$ is true iff $\operatorname{card}(\mathrm{X} \cap \mathrm{Y})=\mathbf{c}$ are atoms of $\mathrm{CINT}$ and functions $\mathbf{g}_{\mathbf{c}}$ such that $\mathbf{g}_{\mathbf{c}}(\mathrm{X})(\mathrm{Y})$ is true iff $\operatorname{card}(\mathbf{X}-\mathbf{Y})=\mathrm{c}$ are atoms of CC-INT.

One notices that atoms of CINT and of CC-INT are denoted by determiners occuring in EXCL phrases with a numeral complement. For instance, given the denotation indicated for (14), it is easy to see that (16a) is true iff condition (16b) is satisfied and (17a) is true iff condition (17b) is satisfied:
a. Every student except five danced
b. $\operatorname{card}(S-D)=5$
a. No student except one danced
b. $\operatorname{card}(S \cap D)=1$

In addition to intersective and co-intersective functions representing atoms I will make use of specific (monotone increasing) intersective and co-intersective functions determined by a given property. Thus for any propery $\mathbf{P}$, the function $\mathbf{F}_{\mathbf{P}}$ such that $\mathbf{F}_{\mathbf{P}}(\mathbf{X})(\mathbf{Y})$ is true iff $\mathbf{P} \subseteq \mathbf{X} \cap \mathbf{Y}$ is an intersective function determined by the property $\mathbf{P}$. Analogously the function $\mathbf{G}_{\mathbf{P}}$ such that $\mathbf{G}_{\mathbf{P}}(\mathbf{X})(\mathbf{Y})$ is true iff $\mathbf{P} \subseteq \mathbf{X}-\mathbf{Y}$ is a co-intersective function determined by property $\mathbf{P}$. The property $\mathbf{P}$ which determines the intersective function also determines a specific atom which is contained in that function and which is obviously an element of the algebra of intersective functions. Similarly with the property which determines a property determined co-intersective function.

Intersective and co-intersective functions determined by a property interpret some specific determiners, as will be shown in more detail below, and consequently noun phrases with such determiners. For instance $\mathbf{F}_{\mathbf{P}}(\mathbf{S})$ interprets the noun phrase All (S) which are $\mathbf{P}$. So given the denotations from the examples in (14) the sentence (18a) is interpreted by (18b):

\section{a. All students who are Albanians danced}

b. $F_{A}(S)(D)$

This observation concerning the interpretation of intersective functions determined by a property is related to the fact that they are filter creating functions. It follows from their definition that if $\mathbf{F}_{\mathbf{P}}$ is an intersective function determined by the property $\mathbf{P}$, then for any $\mathbf{X}, \mathbf{F}_{\mathbf{P}}(\mathbf{X})$ is either the empty set or is the filter generated by $\mathbf{X}$. Similarly if $\mathbf{G}_{\mathbf{P}}$ is a co-intersective function determined by $\mathbf{P}$, then $\mathbf{G}_{\mathbf{P}}(\mathbf{X})$ is either empty or is the ideal generated by $\mathbf{P}$.

In addition to intersective and co-intersective algebras I will make use of restricting algebras, i.e. algebras of restricting functions. Such algebras constitute possible denotations of modifiers. In other words modifiers denote arguably only restricting functions. A modifier is a functional expression of category $\mathbf{C} / \mathbf{C}$ for various choices of $\mathbf{C}$. Thus depending on the choice of $\mathbf{C}$ we have modifiers of any category, in particular of NPs and of determiners. Given the categorisation of modifiers they denote functions from $\mathbf{D}_{\mathbf{C}}$ onto $\mathbf{D}_{\mathbf{C}}$ and the set of all such functions with operations defined pointwise constitutes an atomic (and complete) Boolean 
algebra. Now, it is an important empirical fact that not all logically possible functions of this type are denotations of modifiers found in natural language. As Keenan (1983) claims, and he considers this claim as a language universal, all extensional modifiers denote restrictive functions in the following sense: $\mathbf{F}$ is restricting (in the algebra $\mathbf{D}_{\mathbf{C} / \mathbf{C}}$ ) iff for all $\mathbf{X} \in \mathbf{D}_{\mathbf{C}}, \mathbf{F}(\mathbf{X}) \leq \mathbf{X}$. Thus, to take a simple example of adjectives which are modifiers of common nouns, the fact that all (extensional) adjectives are interpreted by restricting functions means that; roughly, an expression which is a modified common noun entails the corresponding common noun: bold teachers are teachers. The case I will consider in some detail is the case of modifiers modifying determiners occuring in EXCL and NCL phrases.

A specific class of restricting functions is the one which I call conjunctive restricting functions. These are, roughly speaking, restricting functions in which definitions occur the (generalized) conjunction and. The simple example is the following: $\mathbf{f}(\mathbf{x})=\mathbf{x} \cap \mathbf{a}$, where $\mathbf{a}$ is a fixed element of the corresponding algebra. In the analysis I will present I will make use of various, more complex, conjunctive intersecting functions.

The Boolean complement $\mathbf{f}^{\boldsymbol{c}}$ of a restricting function $\mathbf{f}$ is not defined pointwise, but in the following way: $\mathbf{f}^{\mathbf{c}}(\mathbf{x})=\mathbf{x} \cap(\mathbf{f}(\mathbf{x}))^{\prime}$, where $(\mathbf{f}(\mathbf{x}))^{\prime}$ is the Boolean complement of $\mathbf{f}(\mathbf{x})$. One notices that the complement defined in this way has an interesting property: any restricting function $\mathbf{f}$ and its complement $\mathbf{f}^{\boldsymbol{c}}$ both entail the argument to which they apply. This fact can be used to define a generalized notion of presupposition, i.e. presupposition of any category (cf. Zuber 1998). A version of such a definition is given in (19):

A functional expression $\mathbf{E}$ of category $\mathbf{A} / \mathbf{B}$ induces a presupposition $\mathbf{T}$ of category $\mathbf{A}$ iff $\mathbf{E}$ is a modified expression denoting the restricting function $F$ such that for any $X \in D_{B}$ we have $F(X) \leq \operatorname{den}(T)$, where $\operatorname{den}(\mathbf{T})$ is the denotation of $\mathbf{T}$

Less formally, a functional expression $\mathbf{E}$ induces a presupposition $\mathbf{T}$ iff $\mathbf{E}$ is a modified functional expression and for all arguments $\mathbf{A}$ of $\mathbf{E}$, all complex expressions $\mathbf{E}(\mathbf{A})$ entail $\mathbf{T}$. In this sense the possesive noun phrases induces the well-known possessive presuppositions: the noun phrase Leo's cats in (20a) induces (20b) as a presupposition since the schema in (20c) entails, roughly speaking, (20b) for all (extensional) verb phrases VP:
a. Leo's cats
b. Leo has cats
c. Leo's cats VP

Since, as I will show, EXCL and INCL phrases are modified expressions, the above definition of presupposition will be applied to the description of presuppositions induced by such phrases.

\section{Exclusion phrases}

Our purpose is now to determine the semantic structure of EXCL and INCL phrases. In this section I will be mainly concerned with the structure and semantics of EXCL phrases. In particular I would like to present this structure in as fine grained a way as possible by considering the grouping of the main components and 
specificity of the semantic types corresponding to these components. As we know on the surface in EXCL and INCL phrases mainly three elements are involved: a binary connective like except or including and two of their arguments. For technical reasons we can assume a binary branching or (one place) function argument constituency, both in syntax and in semantics. So even if from a purely theoretical point of view the grouping and the catgorisaion of components as function expressions or as argument expressions is not important, we have to decide whether connect applies first to its first argument, independently of the whether this argument is a noun phrase or a determiner, and then to its second argument or the other way around.

Roughly speaking, one can distinguish in the literature two approaches in the analysis of EXCL phrases. Keenan (Keenan and Stavi 1986, Keenan 1996) considers that they result from the application of a discontinous determiner to a common noun. Thus All students except Leo is a result of the application of the (discontinous) determiner All...except Leo to the common noun students. Such determiners denote a co-intersective function and consequently the noun phrase corresponding to the EXCL phrase denotes the value of this function at the property corresponding to students. In fact Keenan shows that in general exclusion determiners denote in the algebra of intersective or co-intersective functions (Keenan 1993). Of course his approach is compatible with an approach in which the analysis of exclusion determiners is pushed further showing their syntactic or semantic composition.

Under the second approach, proposed in particular by Moltmann (Moltmann $1995,1996)$ the EXCL phrases result, syntactically, from the application of some functional expressions, in fact modifiers, to quantified NPs. one gets an NP in the form of an EXCL phrase by applying the "complement expression" but/except NP considered as a modifier, to an NP of the form All CN or No CN. So although in general NPs are rarely modified, in this case, according to Moltmann, we have a modification of NPs. Interestingly enough, in order to account for some semantic properties of EXCL phrases Moltmann has to take into account the internal structure of the modified NPs, and in particular the common noun which occurs there.

Notice that if we consider, following Moltmann, that it is the first argument, the quantified NP which is modified by the exclusion complement then the function denoted by this modifier is not a restricting function. This is because (21a) does not entail (21b) and (22a) does not entail (22b):
a. All students except Leo
b. All students
a. No student except Leo
b. No student

I consider this feature as a negative point in Moltmann's analysis.

Before presenting my proposal I would like to consider a quite different possibility, which is that the connective connect is in the scope of the quantifier occuring in the first NP and thus it connects two properties, at least in some cases. Thus in the EXCL phrase given in (23a) the quantifier No of type $<1,1>$ has as its argument the property $\mathbf{X}$ but $\mathbf{A}$ and similarly in (23b) where the quantifier All has as its argument the same "complex" property:

$$
\begin{aligned}
& \text { a. No (X but } A) \\
& \text { b. All (X but } A)
\end{aligned}
$$


Suppose now, and one can find various arguments for such a claim, that the connective but above means and not. Given this supposition we obtain a semantic interpretation of EXCL phrases which deserves some discussion. Indeed, for instance sentence in (24a) is equivalent to the condition in (24b), which in its turn, is equivalent to (24c), expressing the truth conditions of (24a):
a. No (X but $A)(Y)$
b. $X \cap A^{\prime} \cap Y=\varnothing$
c. $\mathrm{X} \cap \mathrm{Y} \subseteq \mathrm{A}$

Similarly concerning EXCL phrases with universally quantified noun phrases: the truth condition of $(25 \mathrm{a})$ would be the one given in $(25 \mathrm{~b})$ :
a. All (X but $\mathbf{A})$
b. $X-Y \subseteq A$

Now the truh conditions thus obtained give just "half" of those we would like to have: instead of the condition $\mathbf{X} \cap \mathbf{Y}=\mathbf{A}$ we obtain $\mathbf{X} \cap \mathbf{Y} \subseteq \mathbf{A}$. This means that under this interpretation the exception clause does not indicate the exact exception but only maximally possible exception: (26a) comes out to be equivalent to (26b):
a. All students but Albanians
b. All students but at most Albanians

It has been sometimes claimed that there might be a difference between exclusion phrases with but and exclusion phrases with except (Hoeksema 1996). The data here are not always clear but one of the differences might be precisely related to the difference of the type just discussed:but-complements induce only a maximally possible exception as in (26b), whereas except-complements specify the "exact" exception.

Notice finally that the truth conditions indicated in (24c) and in (25b) are the right truth conditions if it is assumed that they concern the atomic expressions denoting atoms of intersective and co-intersective algebras respectively.

I am going to sugest that the modifocation also takes place in EXCL (and NNCL) phrases but what is modified is not an NP but a determiner or rather a det. Futhermore, the determiner which is modified occurs as a "logical constituent" in the second argument, or is the semantic second argument of connect. Thus the exclusion determiners that Keenan treats globally are complex modified determiners obtained by a modification of a simpler ones and the modifier is the discontinous expression No...except or All...except.

Of course from a purely formal point of view it is not important how functional dependence is established in a complex expression in which various elements can be considered as arguments or as functions. However, if we consider that it is the expression $\mathbf{E}$ which varies in EXCL phrases of the form NP connect $\mathbf{E}$, then the range of possible arguments of such a function is much greater than if it is the first argument which varies. Indeed, in EXCL phrases the variation of the first argument is limited to just two quantified noun phrases.

We now have to determine the category, the corresponding type and the logical status of the expression E in EXCL and INCL phrases, and possibly the part of it which is modified. In many cases it follows directly from the data that semantically $\mathbf{E}$ is a det. This is obvious in examples in which the exclusion complement is a 
numeral as in (1b) and (16a), or a numeral restricted by a property, as in (3b) and (9a). Since numerals denote cardinal intersective or co-intersective functions, the expression $\mathbf{E}$ in all these cases is semantically a det.

In order to determine the semantic status of $\mathbf{E}$ when it corresponds to an adjective, a proper noun or a conjunction of proper nouns let us consider the following examples:
a. No student except Leo
b. No student except the student who is Leo
a. No students except Albanians
b. No students except the students who are Albanians
a. Most students, including Leo and Lea
b. Most students including every student who is Leo or Lea
a. Every student except Leo and Lea
b. Every student except the students who are either Leo or Lea

One observes that in these examples phrases in (a) are equivalent to those in (b) and that the complements of exclusion or inclusion clauses in (b) are always full NPs. They are composed of two parts: a common noun, which is the same as the one occuring in the first NP in the above ECXL or INCL phrases (student(s)) and a determiner, often discontinous. All determiners denote property determined dets. In (27a) the denoted det is an intersective function determined by the property corresponding to the singleton containing as the only element Leo and in (28b) the property determining the interpreting intersective function is the property denoted by Albanians. The case in (29b) and (30b) is a bit different: the determining property is the union of individuals denoted by each member of the conjunction of proper nouns occuring as the complement of inclusion clauses in (29a) or as a complement of the exclusion phrase in (30a). For this reason the conjunction in ellided forms in (29a) and (30a) is replaced by a disjunction in full non-elliptic forms in (29b) and (30b).

The examples above do not exhaust all the cases of possible EXCL phrases since the expression $\mathbf{E}$ in such phrases can be also a bare numeral as in (31a). Now, it seems to me, that the meaning of (31a) is the one corresponding to ( $31 \mathrm{~b})$ and not the one in (31c) and even less the one which would be (31d) if it were possible:
a. No/every student except five
b. No/every student except exactly five
c. No/every student except at most five
d. *No/every student except at least five

In other words the numeral complement in EXCL phrases specifies the exact number constiting the exception and not only the least or upper bound for such an exception. This means that the expression $\mathbf{E}$ in such a construction is interpreted by cardinal intersective or cardinal co-intersective functions which in addition are atoms. For the unicity of the proposal to be made we can consider that these functions are also determined by a property, namely by the universal property, the unit of the algebra of properties.

So the picture is the following: we have a large set INTP of intersective or cointersective functions determined by a property, which are candidates for the second semantic argument of the connective connect. This connective first applies to a QNP which is its first semantic argument. By this application one obtains a modifier which modifies the expressions denoting elements of the set INTP and 
as a result of this modification we get determiners forming EXCL or INCL phrases. The types of phrases obtained in this way obviously depends on the choice of connect and, furthermore, of its first argument. If the connective is except and the QNP is formed from No or from All/every, then the phrase thus obtained is an EXCL phrase. The use of the inclusion connective including gives rise to the INCL phrase.

The above discussion leads directly to the semantic description of EXCL phrases. Since the second semantic argument of the connective connect, when it is an exclusion connective, is always a det determined by a property, the EXCL phrases now have one of the two following forms:

$$
\begin{aligned}
& \text { a. No }(X) \text { except } D_{P} \\
& \text { b. Every }(X) \text { except } D_{P}
\end{aligned}
$$

The property $\mathbf{P}$ in the det $\mathbf{D}_{\mathbf{P}}$ above is usually explicitly given. When it is not, as in the case of determiners expressed by bare numerals, it will be the universal property, the unit of the algebra of properties. Given this precision we have the following description of EXCL phrases: the truth conditions of (32a) are given by (33a), which is equivalent to (33b) and those of (32b) are given by (34a), which is equivalent to (34b):
a. $\operatorname{Nop}^{\prime}(X)$ and $D_{P}$
b. No $\left(X \cap P^{\prime}\right)$ and $D_{P}$
a. Every $P^{\prime}(X)$ and $D_{P}-$ not
b. Every $\left(X \cap P^{\prime}\right)$ and $D_{P}-$ not

According to this description (27a) means what is roughly indicated in (35a) and (30a) means what is roughly indicated in (35b). These latter phrases are equivalent respectively to (27b) and (30b) which is what one would like to have:

a. No student who is not Leo and the student who is Leo

b. Every student who is not Leo or Lea and (the students who are Leo or Lea)-not

It might be interesting to see how the above description works in the case of bare numeral exclusion complements. As I said in this case property $\mathbf{P}$ is the universal property 1 . So in this case the first conjunct of the (generalized) conjunctions in (33) and (34) becomes what is given in (36a) and (36b) respectively, where $\varnothing$ is obviously the complement of $\mathbf{1}$ :
a. $\mathbf{N o} \varnothing(\mathbf{X})$
b. Every $\varnothing(\mathbf{X})$

It is easy to see that the expressions in (36a) and (36b) correspond to the unit elements in the algebras of intersective and co-intersective functions respectively. In other words propositions given in (37) and (38) are true for all values $\mathbf{X}$ and $\mathbf{Y}$ :

$$
\begin{aligned}
& \operatorname{No} \varnothing(\mathbf{X})(\mathbf{Y}) \\
& \operatorname{Every} \varnothing(\mathbf{X})(\mathbf{Y})
\end{aligned}
$$


It is a property of Boolean operations that the meet of its unit with any element is equal to this element. So in the case we are discussing we get as the semantic value of EXCL phrases with a bare numeral as an exclusion complement only the second member of the conjunction in (33) and (34), which is the second semantic argument of such phrases. But this is the right result given the fact that we interpret it as an atom of the cardinal intersective or co-intersective algebra.

We can illustrate informally one part of the above discussion just by noting that the sentence in (39) is equivalent to what is in (39b):

a. No student except five is dancing

b. No non-existing (object) except exactly five students is dancing

\section{Inclusion phrases}

Before providing the semantics of INCL phrases I would like to discuss briefly one problem related to their interpretation. It concerns the so-called strict vs non-strict inclusion interpretation of INCL phrases. Suppose we interpret the connective including by the meet in the Boolean algebra of dets. In other words, suppose that $D_{1}$ including $D_{2}$ is interpreted by $D_{1}$ and $D_{2}$, where and is the meet operation in the algebra of dets. Consider now sentences in (40)

a. Some students, including two Albanians, went to the movie

b. Some students, including Leo and Lea, went to the movie

Sentence in (40a) would be true in a model in which there are exactly two students both of them Albanians and both of them went to the movie. Similarily (40b) would be true if Leo and Lea are the only persons who are students and who went to the movie. It does not seem to me that this is the right reading of (40). This way of interpreting the inclusion connective does not account for the fact that in many cases the universe to be considered when interpreting INCL phrases should contain more elements then the universe one considers when interpreting the second argument of the inclusion connective. In other words the "inclusion" in (most) INCL phrases is the strict inclusion, in the set-theoretical sense. I do not have particular empirical arguments to prefer such an interpretation. We will see, however, that if this "strict inclusion" interpretation is accepted (which is possible in all cases of INCL phrases, whereas "non-strict inclusion" interpretation is not always possible) we obtain an interesting semantic relationship between EXCL and the corresponding INCL phrases.

The strict inclusion interpretation is a particular case of the existential import of positive universal or negative universal quantifiers. Notice for instance that the negative universal det No is a particular case of exceptive dets with the "zero exceptions" and it denotes an intersective function in the same way as the dets with non-trivial exceptions. Similarly the det Every, which can be considered as exceptive with "zero exceptions", denotes a co-intersective function in the same way as other exceptive detes with non-trivial exceptions. In this sense No...except $\mathbf{E}$ dets are generalized existential quantifiers and Every...except E are generalized universal quantifiers (cf. Keenan 1993). Now, the interpretation of positive and negative universal quantifiers ("without exceptions") often involves the so-called "existential import", that is the assumption that the universe over which the quantificatikon takes place is not empty. For instance it is in some way assumed that both (41a) and (41b) "imply" that there are some students (at least 
one), since, if not, these sentences would be trivially true, given the "logical" semantics of the quantifiers involved:

a. No student is dancing

b. Every student is dancing

Now we can see why the problem of strict inclusion interpretation is a particular case of the interpretation assuming the existential import: in the above sentences we have phrases with "empty exceptions" and empty sets are strictly included only in non-empty sets.

We see by the same token that the problem of strict inclusion can arise also in connection with EXCL phrases: the set of objects constituting the exception should be strictly included in the set of objects over which the quantificaion goes. Thus one would be tempted to say that (42a) "implies" that there are more than five students and that (42b) "implies" that Leo is not the only student:

a. Every student except five is dancing

b. No student except Leo is dancing

Recall, however, that no assumption implying the existential import has been made in my analysis of ECXL phrases. So, in part for simplicity, I consider that the strict inclusion desiderata is a pragmatic requirement in the case of EXCL phrases but a semantic property in the case of INCL phrases.

There are other reasons for not interpreting the connective including by and applied directly to its arguments. This becomes clear in particular in the case of INCL phrases with a numeral determiner as first argument. Thus if (43a) were interpreted by (43b), we would get a wrong result:

a. Five students, including Leo, were at the movie

b. Five students and the student who is Leo, were sleeping

Indeed, (43a) should be interpreted as entailing that there were exactly five students at the movie whereas (43b) could be true even if Leo was the sixth student.

So the semantic description of INCL phrases should entail their strict-inclusion reading. This roughly means that $\mathrm{NNCL}$ phrases should not only entail their second argument but in addition have an entailment indicating precisely that not only the second argument is entailed. For instance sentences (44a) should entail (44b):

a. Some students, including Leo, went to the movie

b. Not only the student who is Leo, went to the movie

Thus one way to secure the strict-inclusion reading requirement is to use the modifier not-Only, or its equivalent Also, appropriately categorized. Now there is an obvious relation between Only modifying dets and EXCL phrases. To see this in more detail it is preferable to refer to the analysis presented above.

In the analysis of EXCL phrases I used conjunctive restricting functions to interpret modifiers creating these phrases. There are two types of such modifiers: the one based on $N o$ and denoting the restricting function No...except and the modifier based on Every and which denotes the restricting function Every...except. The function No...except applies to intersective functions determined by a property and the function Every...except applies to cointersective functions determined by a property. In addition, in both cases, the application of these functions gives particular atoms determined by the property 
which determines the argument. Now it can be seen that the modifier based on No is semantically equivalent to the lexicalized modifier Only when the latter applies to determiners. In other words phrases No (X) except $\mathbf{P}$ and $\mathbf{O n I y}\left(\mathbf{F}_{\mathbf{P}}(\mathbf{X})\right)$ semantically equivalent. For instance sentences in (45) and in (46) are equivalent:

a. No student(s) except Leo/Albanians passed the examination

b. Only the student(s) who is/are Leo/Albanians passed the examination

a. No students except two went to the movie

b. Only two students went to the movie

As indicated above, in order to assure the strict inclusion reading of INCL phrases we need to use the modifier denoting the restricting function not-Only (Only') which I will denote by Also. Since we know now how Only is defined - it is No...except defined in the preceeding section, and how to negate restricting functions, we can define Also; its definition is given in (47):

$$
\operatorname{Also}\left(F_{A}\right)=F_{A} \text { and }\left(\operatorname{Only}\left(F_{A}\right)^{\prime}\right.
$$

According to the form of the first argument we distinguished two modifiers in EXCL (modifying their second argument): the one based on No and the one based on Every. Since one finds in INCL phrases many more possible first arguments, we have to distinguish more modifiers in this case. For reasons in part related to the discussion of the example (43) we have to distinguish two types of such modifiers in INCL phrases. First, those which correspond to INCL phrases which begin with numeral determiners, and, second, those which correspond to INCL phrases which begin with other types of determiners. Although strictly speaking such a distinction may be not necessary, I provide below semantics for both types of modifiers.

Let me start by INCL phrases beginning by a non-numeral determiner. They have the form given in (48), where $\mathbf{D}$ can be substituted by All, Most Some and At least for instance:

$$
\mathbf{D}(\mathbf{X}) \text { including } \mathbf{E}_{\mathbf{P}}
$$

Recall that there also various restrictions on what can stand for $\mathbf{E}_{\mathbf{P}}$ in the above schema. In particular $\mathbf{E}_{\mathbf{P}}$ cannot be a bare numeral, as opposed to the case of EXCL phrases. This means that from the semantic point of view, $\mathbf{E}_{\mathbf{P}}$ denotes $\mathbf{F}_{\mathbf{P}}$, an intersective function determined by a property $\mathbf{P}$. Phrases of this form have their semantic value given in (49a) which is equivalent to (49b):

$$
\text { a. } \mathbf{D}(\mathbf{X}) \text { and }\left(\operatorname{Also}\left(F_{P}\right)(X)\right.
$$

b. $D(X)$ and $F_{P}(X)$ and $(N o(X) \text { except } P)^{\prime}$

According to this description and the definition of Also given in (47) the phrase in (50a) entails (50b) and (50c):
a. All/some/most students, including Leo
b. the student who is Leo
c. Other students than Leo

When applied to INCL phrases beginning by Most my description gives rise to a particular consequence. Thus the phrase in (51a) entails (51b) 
a. Most students, including Leo, are dancing

b. Most students are dansing

This means that the majority of the students who are dancing may be already constituted without Leo. I do not know whether this is a happy consequence from the empirical point of view. A similar remark can be made with respect to the INCL phrases beginning with the determiner at least.

Concerning the INCL phrases beginning with a bare numeral we have to take care of the problem mentioned in connection with example (43): the cardinality of the objects having the predictated property should not wrongly augment because of the use of the conjunction and. This can be avoided by modifying the initial numeral with the modifier denoting Only. This leads to the following description: INCL phrases of the type given in (52) have their semantics in (53):

$$
\begin{aligned}
& \mathrm{C}(\mathrm{X}) \text { including } \mathrm{E}_{\mathbf{P}} \\
& (\mathrm{Only}(\mathrm{C}))(\mathrm{X}) \text { and }\left(\mathrm{Also}\left(\mathrm{F}_{\mathrm{P}}\right)\right)(\mathrm{X})
\end{aligned}
$$

Thus the semantics of (54a) is given in (54b), supposing that Five denotes Five, students denotes $\mathbf{S}$, danced denotes danced and Leo denotes $\mathbf{L}$ :

a. Five students, including Leo, danced

b. (Only five(S) and Also (the(S)who is L)(danced)

The formula in (53) gives the "exact-reading" of the initial numerals. If for some reason it appears that it is preferable to give for them the "at least-reading" then the formula in (49) can be considered as applying also to INCL phrases with such numeral first arguments.

Let me conclude this section with a brief analysis of negative $\mathrm{N} C L$ phrases. For this purpose we also need an analysis which entails their strict-inclusion reading. Thus we want (55a) to entail (55b):

a. No student, not even Leo

b. Other students than Leo did not (as well)

This reading can be secured by applying the modifier Also to the second argument of the negative INCL phrase and taking its post-negation. So if the negative INCL phrases have the form given in (56), where $\mathbf{N}$ is a determiner which can occur in initial position in such phrases, then their semantics is given in (57a) which, given the equivalence via post-negation between intersective and co-intersective functions, is equivalent to the more explicit formula given in (57b):

$\mathbf{N}(\mathbf{X})$, not even $\mathbf{E}_{\mathbf{P}}$

a. $\mathbf{N}(\mathbf{X})$ and $\left(\left(\operatorname{Also}\left(\mathbf{F}_{\mathbf{P}}\right)\right.\right.$-not $)(X)$

b. $N(X)$ and $F_{P-n o t}$ and $(\operatorname{Every}(X) \text { except } P)^{\prime}$

As an illustration of this analysis we observe that (58a) entails (58b) and (58c):

a. No students, not even Albanians, are in the library

b. No Albanian student is in the library

c. There are other students then Albanians and they are not in the library

The above analysis obviously does not account for the necessary presence of the modifier even in negative INCL phrases, still less for the pragmatic contribution of 
this item. It is interesting, however to notice that even induces some semantic aspects of meaning which support the strict-inclusion reading of negative INCL phrases.

\section{Conclusions}

Using various tools from the algebraic semantics I have proposed a compositional analysis of EXCL and INCL phrases. Both types of phrases are considered as complex NPs formed by the applications of a complex exclusion or inclusion determiner to a common noun phrase. Those determiners in their turn are modified determiners and the way in which they are modified is exhibited. Syntactically the part which is modified at this last stage is a remnant of an ellipsis which is expressed mainly by a proper noun, by an adjective or by a bare numeral. This expression can also be considered as the second argument of the exclusion or inclusion connective and which, except when it is a bare numeral, denotes a property. This means that semantically this second argument can be reconstituted as an intersective or a co-intersective function determined by the property denoted by the surface second argument. In the case of EXCL phrases there are two types of modifiers; one based on No and one based on Every denoting restricting functions No...except and Every...except respectively. The application of these functions to the second argument of the exclusion phrases gives an atom determined by the same property by which the second argument is determined.

The fact that atoms are involved in the proposed analysis has various interesting consequences concerning the relationship between EXCL and the corresponding INCL phrases. Thus it follows from the descriptions given in (33),(49), (53) that all INCL phrases with $\mathbf{E}$ as the second argument entail EXCL phrases with the same $\mathbf{E}$ as second argument but in which the modifier is negated. More precisely phrases of the form (59a) entail, under the srtict-inclusion reading and for any possible determiner $\mathbf{D}$, phrases of the form (59b):

a. $\mathbf{D}(\mathbf{X})$ including $\mathbf{E}$

b. (No(X) except $)^{\prime} \mathrm{E}$

Similarly with negative INCL phrases: given (34) and (57), they all entail the corresponding EXCL phrase in which the negation of the modifier based on Every is used. Thus (60a) entails (60b) for any determiner $\mathbf{N}$ which can occur in negative INCL phrases:

a. $\mathbf{N}(\mathbf{X})$, even not $\mathbf{E}$

b. (Every(X) except)' E

So positive INCL phrases are related to EXCL phrases beginning with No and negative INCL phrases are related to EXCL phrases beginning by Every. More precisely, since negations of modifiers giving rise to atomic expressions entail "coatomic" expressions (i.e. atomic expressions in which the modifier is negated), all positive INCL phrases entail the corresponding co-atomic expressions denoting coatoms of the intersective algebra, and all negative INCL phrases entail the corresponding co-atomic expressions denoting co-atoms in the algebra of cointersective functions.

Furthermore, since complex modified expressions induce in general specific presuppositions, it also follows from the above proposal that sentences with 
EXLC and INCL phrases have particular presuppositions induced by these phrases. Thus it follows from definition (19) that sentences of the form (61a), (61b) and (61c) all presuppose sentences (61d). Similarly (62a) and (62b) both presuppose (62c):
a. No/every student except Leo and Lea VP
b. Most/some/all students including Leo and Lea VP
c. No student not even Leo or Lea
d. Leo and Lea are students
a. No/all students except two passed the examination
b. Most students, including two at the front are sleeping
c. There are at least three students

The last general remark concerning my analysis that I would like to make concerns the type of modifiers which it uses. One notices that in all cases the semantic description given uses basically the generalized conjunction and. In other words the modifiers involved are all conjunctive modifiers, as they were informally characterized. This means that the exclusion and inclusion connectives have both an additive meaning in some sense. I am not going to make this remark more precise. I think, however that this observation should be related to the fact that in many languages the exclusion connectives can also have addirional meaning like English in addition or besides. One can suspect, looking at Kretzman (1982) that some medieval scholars such as Wiliam of Sherwood or Walter Burley associated such an additive meaning with the Latin praete. Kretzman gives the following example of Walter Burley : Some man besides Socrates is risible, which obviously implies an additive meaning of besides. In modern languages this phenomenon is very common: ektos in Grec,ausser(dem) in German or oprocz in Polish can be used as exclusion connectives in EXCL phrases but also as connectives which apply to two NPs with the meaning of in addition (to). In Zuber (forthcoming) the Polish connective oprocz is analysed in this perspective in the framework of algebraic semantics. The description of this connective also involves the notion of atomicity of the corresponding denotational algebras and by the same token relates the analysed phenomenon to other important topics in linguistic semantics.

Given all this, the present contribution should be considered as a modest beginning of work analysing a small part of important specific linguistic data in a rather general and powerful framework of algebraic semantics with a close look at other important phenomena in linguistic semantics such as quantification, focus, ellipsis, conjunction, modification, etc. The results presented here do not make it impossible to hope that unified treatments and various generalisations in semantics are possible and useful.*)

*) Thanks to Ross Charnock who almost voluntarily helped me with some aspects of this paper. He deos not wish to be held responsible for the remaining errors.

\section{References}

von Fintel, K. (1993) Exceptive Constructions, Natural Language Semantics 1, pp.123-148

Hoeksema, J. (1995) The Semantics of Exception Phrases, in van der Does, J. and van Eijck, J. (eds.) Quantifiers, Logic and Language, CSLI Publications, pp.145-178 
Keenan, E. (1983) Boolean Algebra for Linguists, in Morderchay, S. (ed:) UCLA Working Papers in Semantics, pp.1-75

Keenan, E. L. (1993) Natural Language, Sortal Reducibility and Generalized Quantifiers, Journal of Symbolic Logic 58, pp.314-325

Keenan, E.L. (1996) The Semantics of Determiners, in Lappin, S. (ed.) The Handbook of Contemporary Semantic Theory, Blackwell, pp. 41-63

Keenan, E. L. and Faltz, L. M. (1985) Boolean Semantics for Natural Language, D. Reidel Publishing Company, Dordrecht

Kretzman, N. (1982) Syncategoremata, Exponibila, Sophismata, in Kretzman, N. et al: (eds.) The Cambridge History in Late Medieval Philosphy: From the Rediscovery of Aristotle to the Disintegration of Scholasticism 1100-1600, Cambridge University Press, pp. 211-245

Moltmann, F. (1995) Exception Sentences and Polyadic Quantification, Linguistics and Philosophy 18:3, pp.223-280

Moltmann, F (1996) Resumptive Quantifiers in Exception Sentences, in Kanazawa et al. (eds.) Quantifiers, Deduction, and Context, CSLI Publications, Stanford, pp.139-170

Zuber, R. (1997) Some algebraic properties of higher order modifiers, in Becker, T. and Krieger (eds.) Proceedings of the Fifth Meeting on Mathematics of Language, Deutsches Forschungszentrum fur Kunstlische Intelligenz, pp.161-168

Zuber, R. (1998) Constrained Functions and Semantic Information, forthcoming in de Rijke et al. (eds.) Logic, Language and Information, vol.2, CSLI Publications

Zuber, R. (forthcoming) Some categorially polyvalent modifiers in Polish, forthcoming in Proceedings of the Second European Conference on Formal Description of Slavic Languages 